\title{
Multi-input Multi-output Magnetoelectric Dipole Antennas Comprising Dual Monopole Feed Antennas for Energy Harvesting
}

\author{
Wen-Shan Chen, Chien-Min Cheng, ${ }^{*}$ and Guang-Ren Zhang \\ Department of Electronic Engineering, Southern Taiwan University of Science and Technology \\ No. 1, Nan-Tai Street, Yungkang Dist., Tainan City 710, Taiwan R.O.C. \\ (Received June 12, 2018; accepted October 22, 2018)
}

Keywords: multi-input multi-output, energy harvesting, magnetoelectric dipole antenna, electromagnetic band gap

A three-dimensional (3-D) inverted-L multi-input multi-output (MIMO) magnetoelectric dipole antenna comprising two coupled monopole feed antennas is developed for energyharvesting applications. Additionally, the electromagnetic band-gap (EBG) structure was used to improve the isolation $\left(\mathrm{S}_{21}\right)$ of the antennas. The bandwidth of the proposed antenna is up to $42.1 \%$ for $3-4.6 \mathrm{GHz}$, which covers the C-band $(5 \mathrm{G})$ applications of 3.3-3.8 GHz. The advantages of the proposed antenna are the following: low cross-polarization; good radiation patterns; stable and high gains $(9.55 \pm 0.35 \mathrm{dBi})$ in the operating band; all simulated and measured return loss of the antennas $\left(\mathrm{S}_{11}\right.$ and $\left.\mathrm{S}_{22}\right)$ are below $-10 \mathrm{~dB}$; and the isolation between two antennas is below $-15 \mathrm{~dB}$. Finally, the proposed antenna is suitable for modern energyharvesting and 5G wireless sensor network systems.

\section{Introduction}

With the continuous innovation of Internet of Things (IoT) technology and requirement of sensors, for low loss and low environmental pollution of modern electronic products, antennas are used to harvest the free electromagnetic energy in the surroundings, and then, it is converted to electric energy through radio-frequency (RF) circuits. To harvest more electromagnetic energy and from greater distances, antennas with high gains have been developed by many researchers. In 2006, Luk and Wong presented a novel magnetoelectric dipole antenna with a wideband, low cross-polarization, low backlobe, and stable radiation patterns. A threedimensional (3-D) inverted-L dipole antenna was designed and fed by a quarter-wavelength $(\lambda / 4)$ inverted-L probe. As the probe is excited, it will generate a magnetic dipole that combines with the electric dipole generated by the antenna; hence, its impedance bandwidth can be increased effectively. ${ }^{(1)}$ For a simple feed method and better antenna efficiency, the differential feed technique using two symmetric structures was developed for the 3-D inverted-L dipole antenna. The advantages of this feed technique are an increase in the impedance bandwidth, decrease in cross-polarization, and decrease in the backlobe. ${ }^{(2)}$ The reflection phase of the artificial

*Corresponding author: e-mail: ccmin523@gmail.com

https://doi.org/10.18494/SAM.2019.2107 
magnetic conductor (AMC) is 0 and can be put under the loop antenna to decrease the height and increase the gains of the antenna. ${ }^{(3)}$ For the Yagi antenna, the gains for GSM-1800 (1.805-1.88 $\mathrm{GHz})$ and UMTS-2100 (2.11-2.17 GHz) are 10.9 and $13.3 \mathrm{dBi}$, respectively. The energy transform efficiency is as high as $40 \%$, enabling the use of the Yagi antenna in electric energy harvesting. ${ }^{(4)}$ The rectenna has a simple structure, high gain of $8.6 \mathrm{dBi}$, maximum energy transform efficiency of $83 \%$ (operated at $2.45 \mathrm{GHz}$ ), and minimum energy transform efficiency of $50 \%$ even though the input power is only $17.2 \mathrm{dBm}$, making it applicable to energy harvesting. ${ }^{(5)}$ The transform efficiency of energy harvesting could be increased by using high-gain antennas with rectified circuits, solar panels, or folded antennas. ${ }^{(6-9)}$ A wideband dipole rectenna with a vertical metal plate was used to reduce the backlobe to $8.4 \mathrm{~dB}$, which enables an increase in its energy transform efficiency. ${ }^{(10)}$ A differentially fed microstrip antenna with a high gain of 8.5 $\mathrm{dBi}$, high efficiency of $80 \%$, and operated at GSM-900 (900-960 MHz) was presented. It could be applied at the front of the RF circuits and modified the impedance matching for efficiency improvement. ${ }^{(11)}$

To find the best antenna for energy harvesting, the short dipole antenna, patch antenna, and Yagi antenna were compared. It was found that a small antenna could not satisfy the requirements of energy harvesting, but the combination of a Yagi antenna and rectifier yielded much better results than other antennas. ${ }^{(12)}$ In this work, to obtain better antenna efficiencies, gains, and a faster transmission speed of signals, we developed a 3-D inverted-L multi-input multi-output (MIMO) magnetoelectric dipole antenna. On the basis of the idea of the hinge antenna of notebook computers, the feed technique of this proposed antenna was to use two coupled monopole antennas to excite the closed slots located between two hinges. ${ }^{(13,14)}$ These two coupled monopole feed antennas excited the slots and generated the magnetic dipole, which combines with the electric dipole generated by the inverted-L dipole antenna. Two similar beam widths can be obtained for $Y Z$ and $X Z$ planes, and they can cover the modern C-band $(5 \mathrm{G})$ of 3.3-3.8 GHz. Furthermore, we also used the electromagnetic band-gap (EBG) structure to suppress the surface current occurring between two antennas, and finally increased its isolation and reduced the interference to enable energy-harvesting applications. ${ }^{(15-17)}$

\section{Antenna Design}

\subsection{Antenna structure}

A 3-D MIMO magnetoelectric dipole antenna was designed and fabricated on a flame retardant 4 (FR4) substrate with a thickness of $0.8 \mathrm{~mm}$, permittivity $\left(\varepsilon_{r}\right)$ of 4.4 , and loss tangent $(\tan \delta)$ of 0.0245 . The proposed antenna was composed of an $85 \times 85 \mathrm{~mm}^{2}$ metal reflector, four polyactide (PLA) pillars, a $42 \times 17 \mathrm{~mm}^{2}$ metal ground, an EBG structure, two coupled monopole feed antennas, and a pair of 3-D inverted-L dipole antennas, as Fig. 1 shows. Additionally, four PLA pillars were added between the ground and the reflector. They were made using a 3-D printer and had a height of $15 \mathrm{~mm}, \tan \delta$ of 0.25 , and $\varepsilon_{r}$ of 4 . Two inverted-L dipole antennas were excited by two coupled monopole antennas resonated at $3.65 \mathrm{GHz}$ with an electric length of half the guided wavelength $(\lambda / 2)$. However, on both sides of the metal ground, 


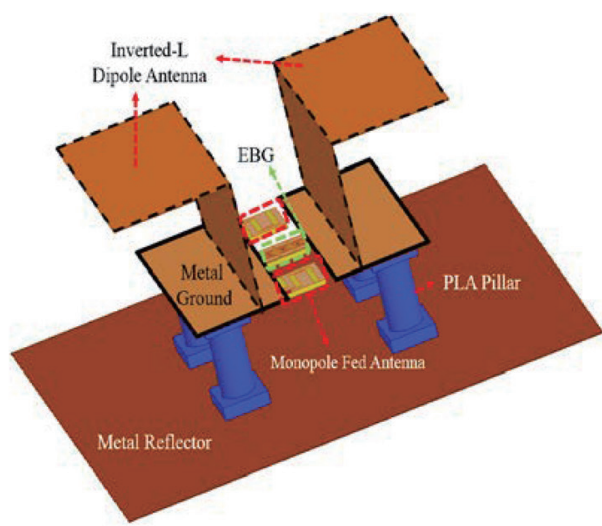

(a)

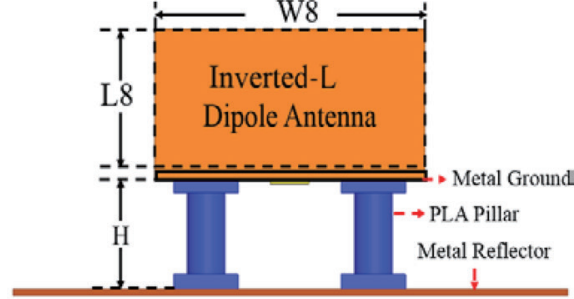

(b)

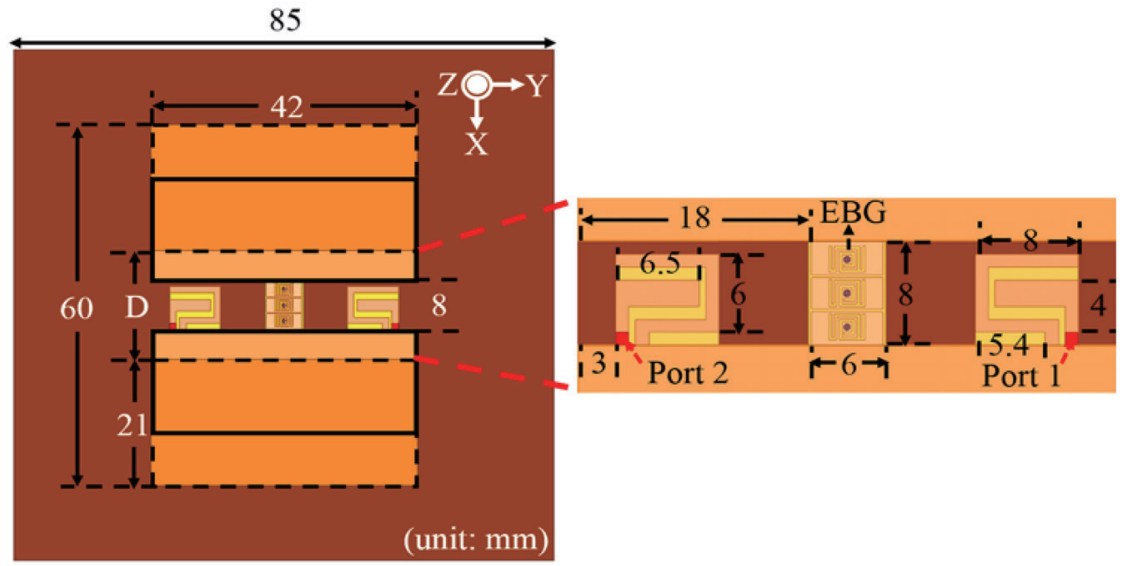

(c)

Fig. 1. (Color online) Geometry of proposed antenna: (a) 3-D, (b) side view, and (c) top view.

two open slots were also excited by these two feed antennas, generating a magnetic dipole that resonated at $4.1 \mathrm{GHz}$ with an electric length of one-quarter the guided wavelength $(\lambda / 4)$ and that combined with the electric dipole generated by the inverted-L dipole antennas. Two similar and stable beamwidths can thus be generated with low cross-polarization for both $Y Z$ and $X Z$ planes. In the previous paper, ${ }^{(18)}$ the authors expanded the applications of the photonic crystals to the microwave field because of its band-gap property, and designed some equivalent circuit structures for generating a band-gap effect of specified frequency. Thus, it was called the EBG structure. As shown in Fig. 2(a), the mushroom-like EBG proposed by Sievenpiper et al. is the most widely studied and discussed. It is an array composed of square metal sheets that are connected to the ground plane through a via located at the center of each metal sheet. This structure will generate capacitive effects owing to the electric field existing among the metal sheets, and the current flow through the via will generate inductive effects owing to the magnetic field. These two effects are equivalent to a parallel LC circuit. ${ }^{(19)}$

In this work, to increase the amount of coupling and generate a band gap at about $2.7 \mathrm{GHz}$, we used FR4 as the substrate and developed a modified EBG structure with three groups of 


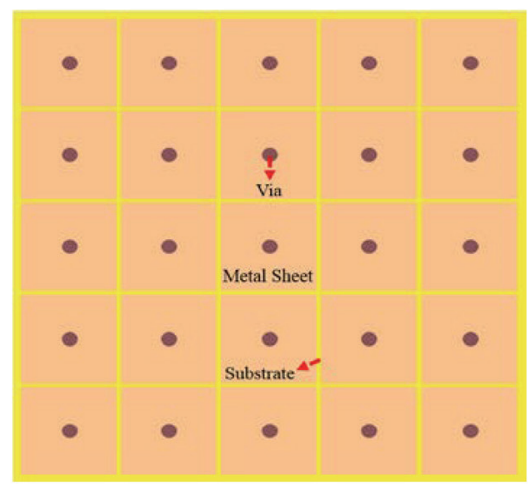

(a)

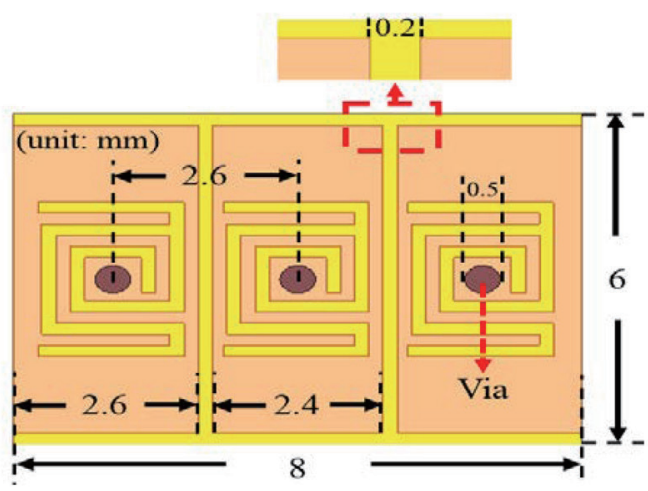

(b)

Fig. 2. (Color online) Geometry of EBGs: (a) mushroom-like EBG ${ }^{(18)}$ and (b) dual-spiral EBG (proposed).

compact dual spirals and vias, as shown in the center of Fig. 1(c) and enlarged in Fig. 2(b). The EBD structure was composed of three dual spirals and connected to the back metal plane through three vias with copper rods. However, two metal grounds are connected by the EBG and thus two open slots existed between them. These slots can increase the equivalent capacitance and the spiral structure can increase the equivalent inductance. ${ }^{(20,21)}$

\subsection{Suspending microstrip line measurement of proposed EBG}

To investigate the isolation influences of the EBG between these two coupled monopole antennas, we adopted the suspended microstrip line measurement to analyze the EBG. In addition, the EBG was sandwiched tightly between two FR4 substrates and connected to the back metal plane through the via, as shown in Fig. 3. Then the microstrip line was fabricated on the upper FR4 substrate and connected to two SubMiniature version A (SMA) connectors. The strong mutual coupling effect between the microstrip line and EBG reduces the parasitic effects generated by other devices, and hence, reveals the band-gap properties. ${ }^{(20,21)}$ The simplified equivalent parallel LC circuit is shown in Fig. 4 and its resonant frequency $\left(f_{s}\right)$ can be derived as Eq. (1), where $L$ is the inductance due to current flows through the via, $C_{1}$ is the capacitance between the upper microstrip line and the EBG, and $C_{2}$ is the capacitance between the EBG and the back metal ground. It can be seen that the increasing capacitance or inductance will cause the decrease in resonant frequency. Additionally, the three dual spirals also generate parts of inductance and capacitance for $C_{1}, C_{2}$, and $L$. The measured and simulated isolation $\left(\mathrm{S}_{21}\right)$ for $2-5 \mathrm{GHz}$ are plotted in Fig. 5. It is found that the EBG reveals better-simulated $\mathrm{S}_{21}$ (lower than $-20 \mathrm{~dB}$ ) at about $2.7 \mathrm{GHz}$. However, the measured $\mathrm{S}_{21}$ shifts to lower frequency and reveals better impedance matching and bandwidth, which is consistent with previous reports. ${ }^{(22,23)}$ Figure 6 shows the $S_{21}$ values with and without (with metal sheet) the EBG. It is seen that without the EBG, the influence between two antennas is serious (greater than $-15 \mathrm{~dB}$ ) for 3.3-4.7 GHz. However, after EBG is used, the resonant frequency of the EBG is shifted 


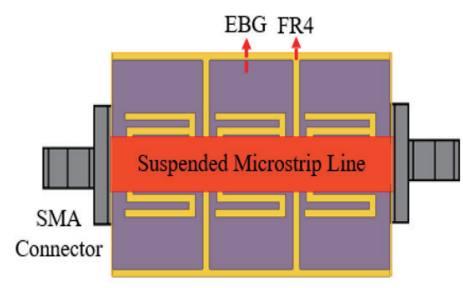

(a)

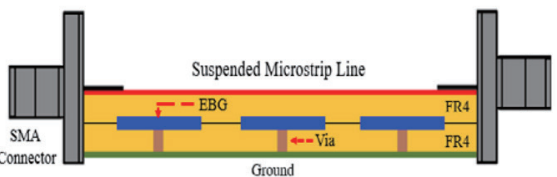

(b)

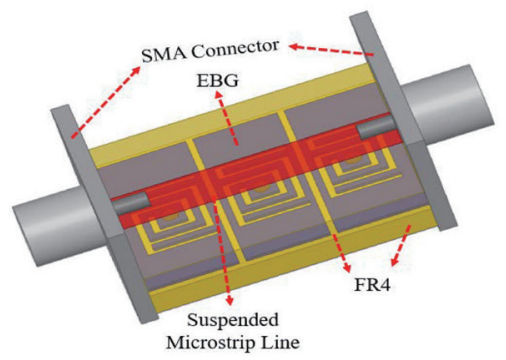

(c)

Fig. 3. (Color online) Suspended microstrip line measurement of EBG: (a) top view, (b) side view, and (c) 3-D view.
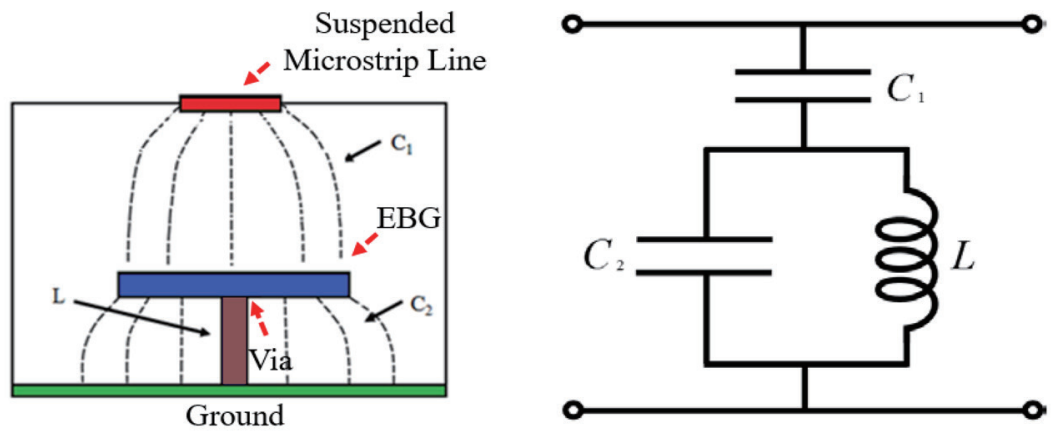

Fig. 4. (Color online) Simplified equivalent circuit of EBG.

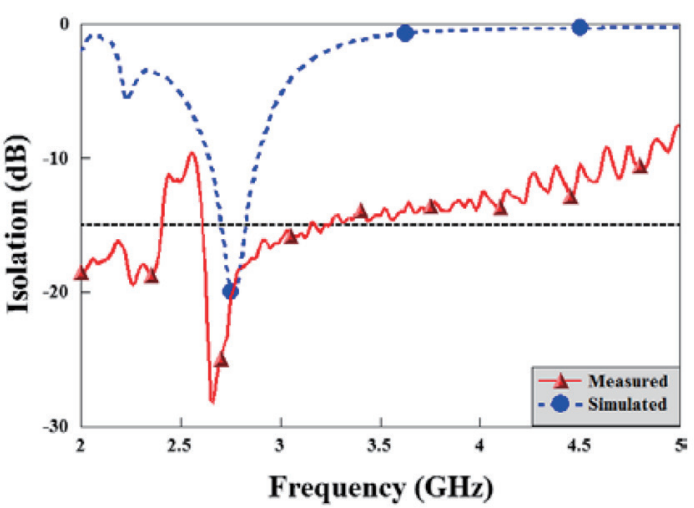

Fig. 5. (Color online) Simulated and measured S parameters of EBG for suspended microstrip line measurement.

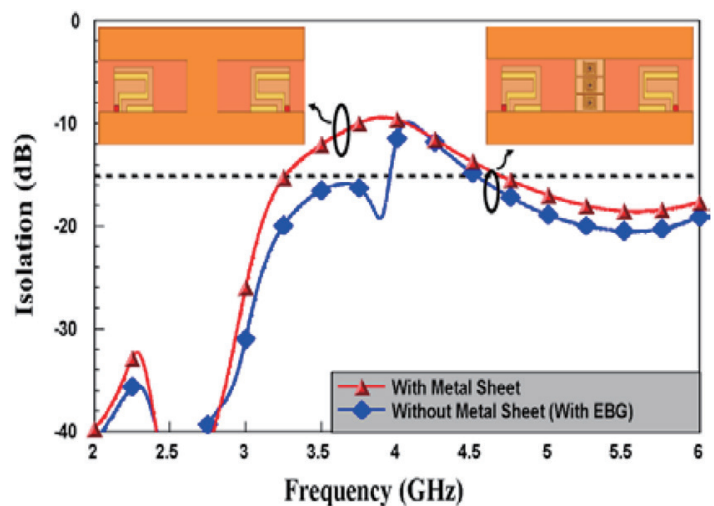

Fig. 6. (Color online) Simulated $\mathrm{S}_{21}$ values with and without metal sheet (with EBG). 
from 2.7 to $3.8 \mathrm{GHz}$, which means that the isolation is modified and better than that without the EBG. Figure 7 shows the current distribution plots in the case of with and without the EBG. Compared with without the EBG, we find that the surface current is obviously suppressed by the use of the EBG and hence, the isolation is improved.

$$
f_{s}=\frac{1}{2 \pi \sqrt{L\left(C_{1}+C_{2}\right)}}
$$

\section{Results and Discussion}

Figure 8 shows the simulated $\mathrm{S}$ parameters for different distances between two inverted-L dipole antennas ( $D$ values) with the PLA pillar height $(H)$ fixed at $15 \mathrm{~mm}$. From the simulated $\mathrm{S}_{11}$ and $\mathrm{S}_{22}$ curves shown in Fig. 8(a), with $D$ values equal to $14-18 \mathrm{~mm}$, all the $\mathrm{S}$ parameters are below $-10 \mathrm{~dB}$ for $3.3-4.4 \mathrm{GHz}$. This means that the excitation wavelengths of two coupling feed antennas will decrease owing to the increase in the distance between two inverted-L dipole antennas. In addition, the first resonated mode (at about $3.65 \mathrm{GHz}$ ) and the second resonated mode (at about $4.1 \mathrm{GHz}$ ) are all shifted to higher frequencies as $D$ values are decreased. Figure 8(b) shows $\mathrm{S}_{21}$, and we can see that as $D$ values are decreased, the two inverted-L antennas will

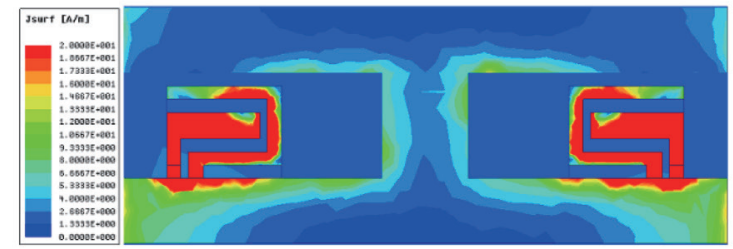

(a)

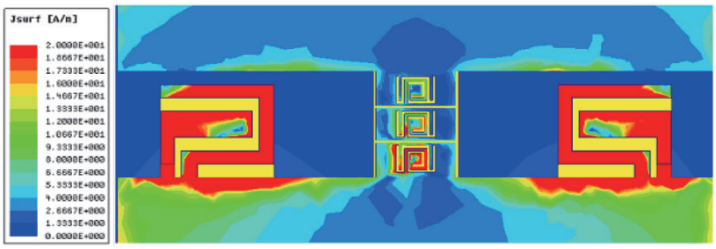

(b)

Fig. 7. (Color online) Simulated 3.8 GHz current distribution plots: (a) with metal sheet and (b) without metal sheet but with EBG.

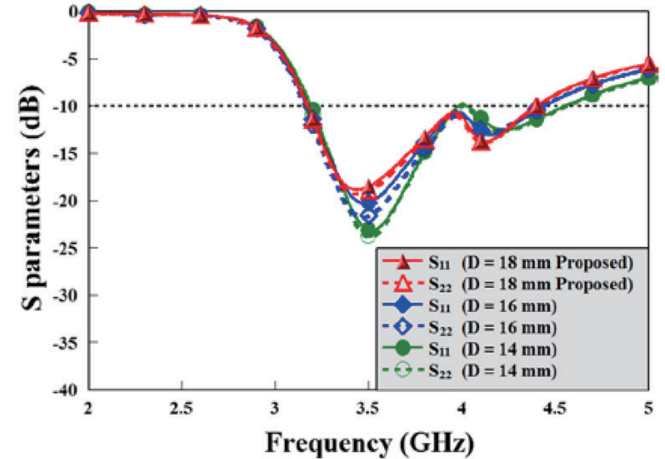

(a)

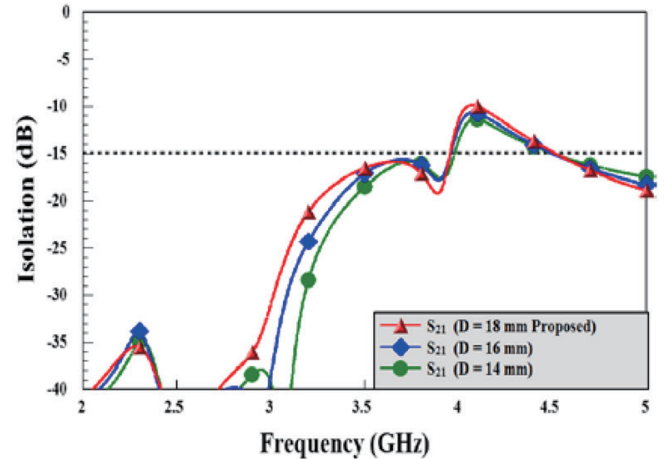

(b)

Fig. 8. (Color online) Simulated $\mathrm{S}$ parameters for different $D$ values $(H=15 \mathrm{~mm})$ : (a) $\mathrm{S}_{11}$ and $\mathrm{S}_{22}$ and (b) $\mathrm{S}_{21}$. 
become closer to the two feed antennas. Thus, the isolation will gradually worsen. Figure 9 shows the simulated antenna gains for different $D$ values ( $H$ is fixed at $15 \mathrm{~mm}$ ). It can be seen that for the operation band (3.3-3.8 GHz), the antenna gains clearly decrease with decreasing $D$ values. This phenomenon is caused by the variation of the radiation patterns, which is induced by the alterations of the electric and magnetic dipoles. Additionally, as the $D$ value is $18 \mathrm{~mm}$, we can obtain high antenna gains easily and with the maximum gain of $10.4 \mathrm{dBi}$ (at $3.5 \mathrm{GHz}$ ) and the minimum gain of $9.9 \mathrm{dBi}$ (at $3.8 \mathrm{GHz}$ ).

For the distance between two inverted-L dipole antennas fixed at $18 \mathrm{~mm}$, Fig. 10 shows the simulated return loss for different $H$ values $(13,14$, and $15 \mathrm{~mm})$. We find, from the simulated return loss shown in Fig. 10(a), that all the $S_{11}$ and $S_{22}$ values are below $-10 \mathrm{~dB}$ for 3.3-4.4 GHz. This means that all resonated modes of the antenna were affected by the air thickness and the heights of the PLA pillars. Furthermore, we can see from the $S_{21}$ plots shown in Fig. 10(b) that, as $H$ values decrease, the metal reflector will become closer to the metal ground, and

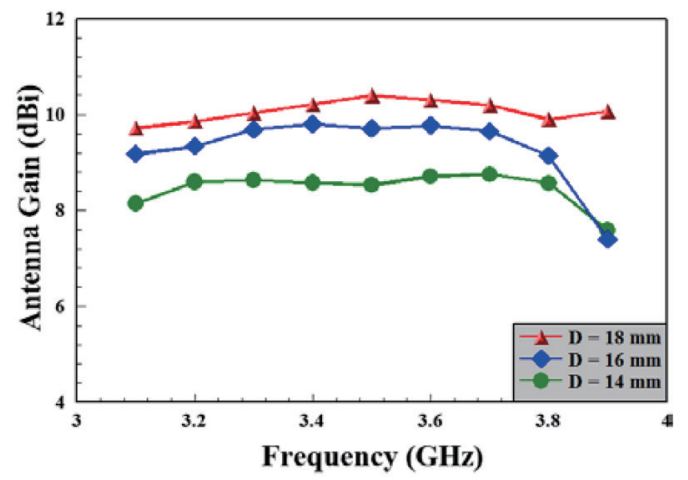

Fig. 9. (Color online) Simulated antenna gains for different $D$ values $(H=15 \mathrm{~mm})$.

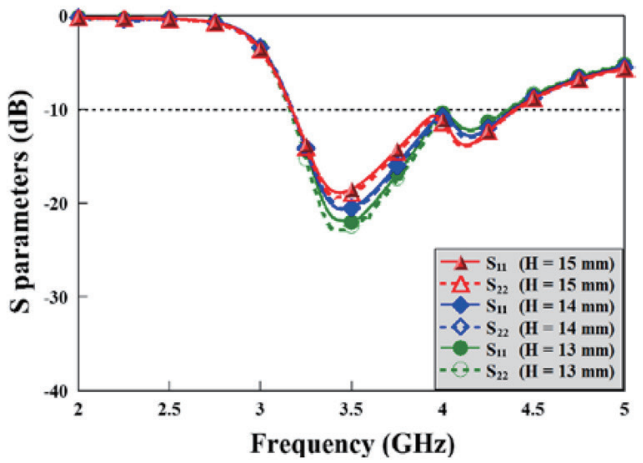

(a)

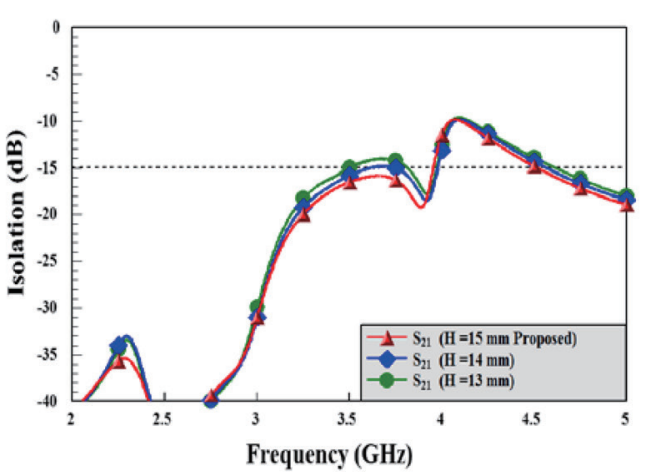

(b)

Fig. 10. (Color online) Simulated $\mathrm{S}$ parameters for different $H$ values $(D=18 \mathrm{~mm})$ : (a) $\mathrm{S}_{11}$ and $\mathrm{S}_{22}$ and (b) $\mathrm{S}_{21}$. 
thus the isolation will gradually worsen (greater than $-15 \mathrm{~dB}$ at $H=14 \mathrm{~mm}$ ). Figure 11 shows the simulated antenna gains for different $H$ values ( $D$ is fixed at $18 \mathrm{~mm}$ ). It can be seen that for the operation band of 3.3-3.8 GHz, the antenna gains decrease with decreasing $H$. This phenomenon may be caused by the variation in the impedance matching owing to different $H$ values. Additionally, when the $H$ value is $15 \mathrm{~mm}$, the maximum gain is $10.4 \mathrm{dBi}$ (at $3.5 \mathrm{GHz}$ ) and the minimum gain is $9.9 \mathrm{dBi}$ (at $3.8 \mathrm{GHz}$ ).

For $H$ of $15 \mathrm{~mm}$ and $D$ of $21 \mathrm{~mm}$, the simulated and measured $\mathrm{S}$ parameters $\left(\mathrm{S}_{11}, \mathrm{~S}_{22}\right.$, and $\mathrm{S}_{21}$ ) of the proposed antenna are shown in Fig. 12. It is found that for the operation band of 3.3-3.8 GHz, both the simulated and measured return losses $\left(\mathrm{S}_{11}\right.$ and $\left.\mathrm{S}_{22}\right)$ are lower than $-10 \mathrm{~dB}$. In addition, all the measured $\mathrm{S}_{21}$ values are lower than $-15 \mathrm{~dB}$. Compared with the simulated impedance bandwidth of $32.5 \%$ (3.17-4.4 GHz), the measured impedance bandwidth (3-4.6 GHz) increased by about $9.6 \%$ to $42.1 \%$. Owing to fabrication errors and the use of the EBG, the resonant frequency shifted to a lower frequency but was still within the range of C-band (5G) applications of 3.3-3.8 GHz. Figure 13 shows the simulated and measured 2-D $Y Z$ - and XZ-plane radiation patterns of the proposed antenna for 3.3, 3.4, 3.5, and 3.6 GHz. We find, from the figure, that both the simulated and measured radiation patterns reveal similar beamwidths in both the $Y Z$ and $X Z$ planes. This means that this proposed antenna achieves stable and unidirectional radiation patterns and lower cross-polarization.

Figure 14 shows the simulated and measured antenna gains and efficiencies. In the operation band of 3.3-3.8 GHz, on comparing these two figures, we can see that both the simulated results are more stable than the measured one. For the simulated results shown in Fig. 14(a), the gain is stable with a variation of only $4.9 \%$. The minimum value is $9.9 \mathrm{dBi}$ at $3.2 \mathrm{GHz}$ and the maximum value is $10.4 \mathrm{dBi}$ at $3.5 \mathrm{GHz}$. Moreover, the lowest simulated efficiency is $80 \%$ at $3.8 \mathrm{GHz}$, and the highest simulated efficiency is $91 \%$ at $3.3 \mathrm{GHz}$. However, for the measured results shown in Fig. 14(b), the gain is stable with a variation of $7.3 \%$, a minimum value of 9.2 $\mathrm{dBi}$ at $3.2 \mathrm{GHz}$, and a maximum value of $9.9 \mathrm{dBi}$ at $3.5 \mathrm{GHz}$. Moreover, the lowest measured efficiency is $61 \%$ at $3.8 \mathrm{GHz}$, and the highest measured efficiency is $75.8 \%$ at $3.3 \mathrm{GHz}$.

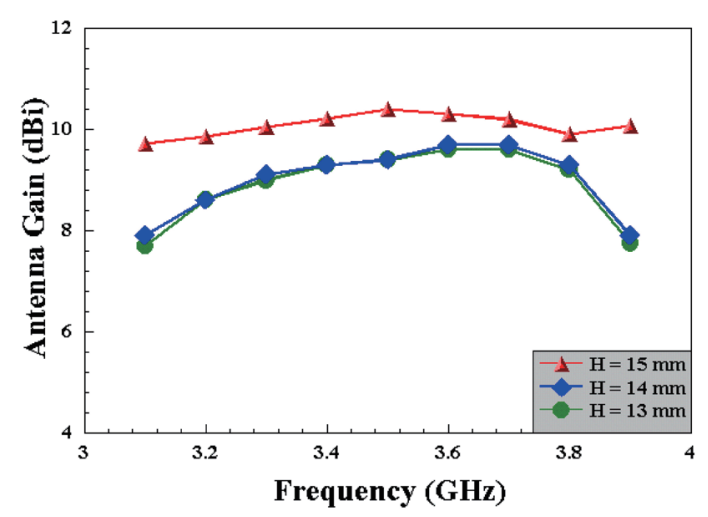

Fig. 11. (Color online) Simulated antenna gains for different $H$ values $(D=18 \mathrm{~mm})$.

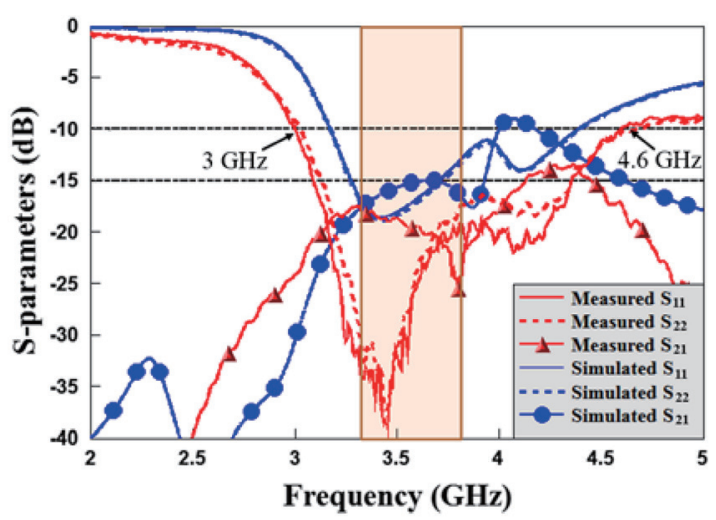

Fig. 12. (Color online) Simulated and measured S parameters $(H=15 \mathrm{~mm}, D=21 \mathrm{~mm})$. 


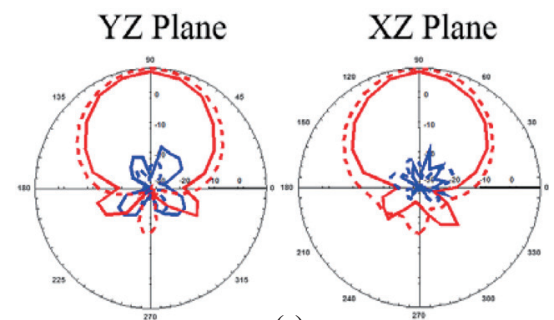

(a)

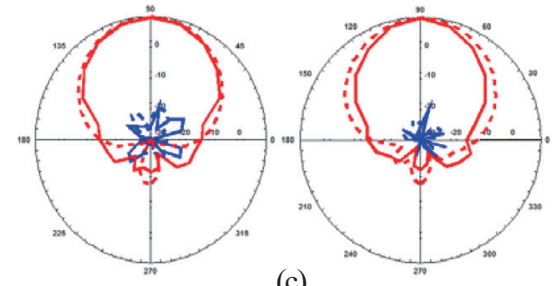

(c)

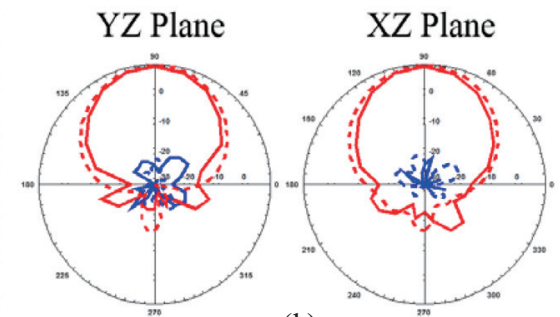

(b)

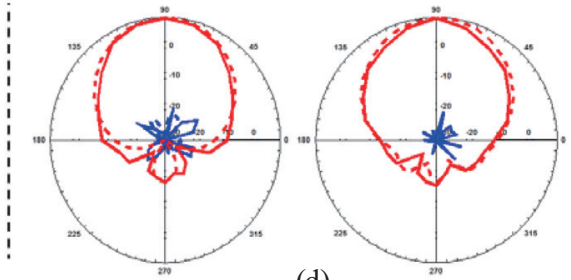

(d)

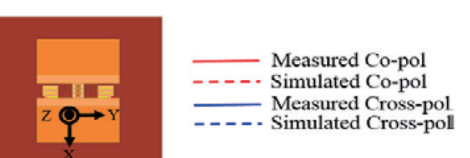

Fig. 13. (Color online) Simulated and measured antenna radiation patterns: (a) 3.3, (b) 3.4, (c) 3.5, and (d) $3.6 \mathrm{GHz}$.

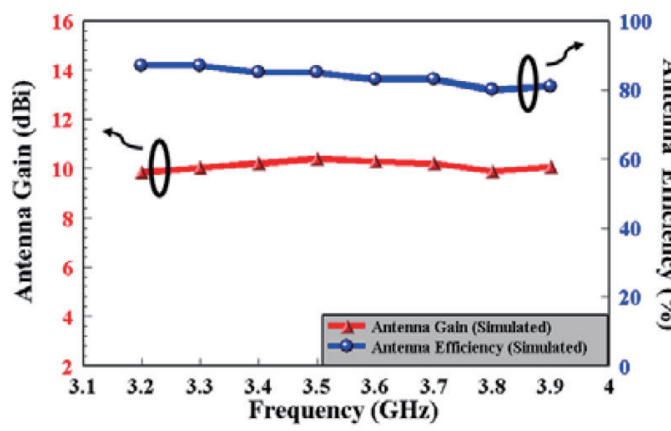

(a)

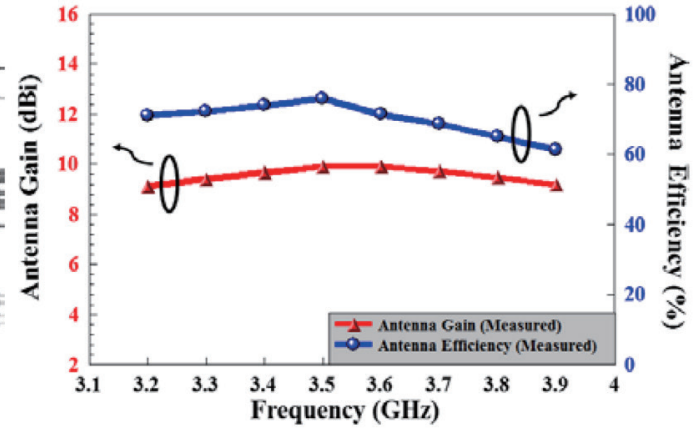

(b)

Fig. 14. (Color online) (a) Simulated and (b) measured antenna gains and efficiencies.

Table 1

Simulated and measured characteristics $(H=15 \mathrm{~mm}, D=18 \mathrm{~mm})$.

\begin{tabular}{llccccc}
\hline $\begin{array}{l}\text { Bands } \\
(\mathrm{GHz})\end{array}$ & & $\begin{array}{c}\text { Max. gain } \\
(\mathrm{dBi})\end{array}$ & $\begin{array}{c}\text { Min. gain } \\
(\mathrm{dBi})\end{array}$ & $\begin{array}{c}\text { Max. efficiency Min. efficiency } \\
(\%)\end{array}$ & $(\%)$ & $\begin{array}{c}\text { Gain variation } \\
(\%)\end{array}$ \\
\hline \multirow{2}{*}{$3.3-3.8$} & Simulated & 10.4 & 9.9 & 90 & 80 & 4.9 \\
& Measured & 9.9 & 9.2 & 75.8 & 61 & 7.3 \\
\hline
\end{tabular}




\section{Conclusions}

With two coupling monopole antennas for excitation and the addition of a metal reflector, a 3-D inverted-L MIMO magnetoelectric dipole antenna with stable radiation patterns and high antenna gains and efficiencies was achieved. The EBG structure was used to suppress the surface current between two dipole antennas and hence increase their isolation. As shown in Table 1, for the C-band (5G) applications of 3.3-3.8 GHz, with $H=15 \mathrm{~mm}$ and $D=18 \mathrm{~mm}$, the optimum properties of the proposed antenna were high stability (simulated variation was $4.9 \%$ and measured variation was 7.3\%), high antenna gains (simulated: $10.4 \mathrm{dBi}$; measured: $9.9 \mathrm{dBi}$ ), high efficiency (simulated: 90\%; measured: 75.8\%), low cross-polarization, return loss below $-10 \mathrm{~dB}$, and isolations below $-15 \mathrm{~dB}$. The proposed antenna is thus suitable for future energyharvesting applications and $5 \mathrm{G}$ wireless sensor network systems.

\section{Acknowledgments}

This work was kindly supported by the Ministry of Science and Technology of the Republic of China (MOST 106-2221-E-218-034).

\section{References}

K. M. Luk and H. Wong: Int. J. Microwave Opt. Technol. 1 (2006) 34.

2 S. W. Liao, Q. Xue, and J. H. Xu: IEEE Antennas Propag. Mag. 55 (2013) 74.

3 H. Kamoda, S. Kitazawa, N. Kukutsu, and K. Kobayashi: IEEE Trans. Antennas Propag. 63 (2015) 4408.

4 H. C. Sun, Y. X. Guo, M. He, and Z. Zhong: IEEE Antennas Wirel. Propag. Lett. 12 (2013) 918.

5 H. C. Sun, Y. X. Guo, M. He, and Z. Zhong: IEEE Antennas Wirel. Propag. Lett. 11 (2012) 929.

6 Y. Tawk, J. Costantine, F. Ayoub, and C. G. Christodoulou: IEEE Antennas Propag. Mag. 60 (2018) 132.

7 S. Karimkashi and A. A. Kishk: IEEE Trans. Antennas Propag. 57 (2009) 3813.

8 F. Xie, G. M. Yang, and W. Geyi: IEEE Antennas Wirel. Propag. Lett. 12 (2013) 155.

9 T. S. Almoneef, H. Sun, and O. M. Ramahi: IEEE Antennas Wirel. Propag. Lett. 15 (2016) 1406.

10 J. I. Moon, I. K. Cho, S. M. Kimand, and Y. B. Jung: Electron. Lett. 49 (2013) 1050.

11 M. Arrawatia, M. S. Baghini, and G. Kumar: IEEE Trans. Antennas Propag. 63 (2015) 1983.

12 P. Soboll, V. Wienstroer, and R. Kronberger: IEEE Microwave Mag. 17 (2016) 75.

13 S. C. Chen and Y. C. Tsou: IEEE Trans. Antennas Propag. 52 (2016) 794.

14 S. C. Chen and Y. C. Tsou: IEEE Trans. Antennas Propag. 64 (2016) 3707.

15 S. D. Assimonis, T. V. Yioultsis, and C. S. Antonopoulos: IEEE Trans. Magn. 48 (2012) 771.

16 G. E. Domínguez, J. M. F. Gonzalez, P. Padilla, and M. S. Castañer: IEEE Antennas Wirel. Propag. Lett. 11 (2012) 1265.

17 S. D. Assimonis, T. V. Yioultsis, and C. S. Antonopoulos: IEEE Trans. Antennas Propag. 60 (2012) 4944.

18 E. Yablonovitch: J. Opt. Soc. Am. 10 (1993) 283.

19 D. Sievenpiper, L. Zhang, R. F. Jimenez Broas, N. G. Alexopolous, and E. Yablonovitch: IEEE Trans. Microwave Theory Tech. 47 (1999) 2059.

20 L. Yang, M. Fan, and Z. Feng: Asia-Pacific Microwave Conf. (APMC) (2005) 4.

21 L. H. Mei, Z. Hua, S. S. Ming, and F. S. Wei: 4th Asia-Pacific Conf. Anten. Propag. (APCAP) (2015) 307.

22 S. Y. Wang and F. C. Chen: 42nd Eur. Microwave Conf. (EuMC) (2012) 663.

23 Z. Yan, Z. Ning-ning, S. Q. Lin, L. S. Wei, and Z. Jun: Int. Conf. Meas. Tech. Mech. Auto. (ICMTMA) (2011) 350 . 